\title{
Evaluation of Pediatric-Specific Wheelchair-Skills-Training Tools By Primary Users: A Descriptive Qualitative Study
}

\author{
Béatrice Ouellet \\ Université Laval \\ Paula W Rushton ( $\sim$ paula.rushton@umontreal.ca) \\ Université de Montréal \\ Andrée-Anne Côté \\ Université de Montréal \\ Laurence Fortin-Haines \\ Université de Montréal \\ Emma Lafleur \\ Université de Montréal \\ Isabelle Paré \\ Université de Montréal \\ Melanie Barwick \\ University of Toronto \\ R. Lee Kirby \\ Dalhousie University \\ Maxime T Robert \\ Université Laval \\ François Routhier \\ Université Laval \\ Yohali Burrola-Mendez \\ Université de Montréal \\ Krista L Best \\ Université Laval
}

\section{Research Article}

Keywords: Occupational therapy, Wheelchair Skills Training Program, Knowledge transfer, Pediatric Rehabilitation, Knowledge-to-action, qualitative study

Posted Date: October 21st, 2021

DOI: https://doi.org/10.21203/rs.3.rs-955768/v1

License: (c) (i) This work is licensed under a Creative Commons Attribution 4.0 International License. Read Full License 


\section{Abstract \\ Background}

Children's ability to engage in meaningful occupations is positively influenced by their ability to move independently. Preliminary evidence in children suggests that wheelchair skills training improves wheelchair skills, which are critical for wheelchair mobility. The Wheelchair Skills Training Program is a standardized program to teach wheelchair skills. However, it is underutilized in pediatric rehabilitation settings. To address perceived barriers to training, three pediatric-specific Wheelchair Skills Training Program tools for indoor skills were developed (i.e., a storybook, four instructional posters, and a workbook). This study aimed to document occupational therapists' and pediatric manual wheelchair users' satisfaction and perspectives on the usability, relevance, and feasibility of these tools.

\section{Method}

A descriptive qualitative research design was used. A focus group was conducted with occupational therapists and interviews were conducted pediatric manual wheelchair users to obtain their feedback on the tool prototypes to facilitate refinement and to ensure they meet users' needs.

\section{Results}

Eight occupational therapists and five pediatric manual wheelchair users expressed general satisfaction with the tools, describing them as usable, relevant and feasible to integrate into wheelchair skills training with novice wheelchair users and younger children. All occupational therapists and three pediatric manual wheelchair users expressed the desire to use the tools for wheelchair skills training. Two children expressed that the tools were more relevant for beginner wheelchair users only. The participants suggested minor modifications to make the tools more accessible for children (e.g., more action in the story, increased precision of illustrations related to the characters' position in the wheelchair).

\section{Conclusion}

Occupational therapists and pediatric manual wheelchair users perceived the tools to have high potential to facilitate the use of the Wheelchair Skills Training Program in pediatric rehabilitation settings. After making the recommended modifications, the tools will be ready for pilot testing in pediatric rehabilitation settings.

\section{Background}

Occupational therapists (OTs) are traditionally responsible for wheelchair skills training. One of the main goals for pediatric manual wheelchair users (PMWUs) is to promote their participation in activities that contribute to their development. Such activities include play, social interaction with peers, exploration opportunities, and independent mobility $(1,2)$. Given that meaningful engagement in occupations is positively influenced by a child's ability to move independently, training of wheelchair skills may facilitate participation and reduce dependency on parents for everyday mobility $(1,3)$.

The Wheelchair Skills Program (WSP) is a standardized program consisting of assessment (Wheelchair Skills Test) and training (Wheelchair Skills Training Program [WSTP]) tools that OTs could use when working with PMWUs $(4,5)$. The WSTP is effective, safe, and practical for improving wheelchair skills and confidence among adult users $(6,7)$. The evidence has been replicated in various clinical populations, in low and high-resourced settings, in a variety of clinical and community contexts and using various training strategies $(6,7)$. In pediatrics, there is preliminary evidence supporting the WSTP for improving wheelchair skills, wheelchair use confidence and participation in meaningful activities when offered in groups and by a peer-trainer $(5,8)$. When administered over two, four hours sessions to a group of PMWUs between 6 and 15 years of age, the WSTP also decreased shoulder pain and increased independence (5).

Although the benefits of the WSTP have been documented, the quantity and quality of wheelchair skills training provided to children appears to be insufficient. Despite OTs perceiving wheelchair skills training to be important, a gap has been identified in the clinical uptake of effective programs $(9,10)$. For example, a survey of 68 Canadian rehabilitation centers (43 of which offered services to children), revealed that most clinicians spent two hours or less training manual wheelchair skills and 18\% provided no training at all (11). Moreover, fewer than $30 \%$ of clinicians used an evidence-based training program and the wheelchair skills training that was provided focused on basic skills (11). Similar results were reported by OTs from a pediatric rehabilitation center and its affiliated schools in Montreal (9). Given that many PMWUs 
have difficulties performing the skills necessary to participate in daily activities $(2,12)$, rely on others for outdoor mobility (2), and have suffered wheelchair-related injuries (13), it is critical to identify strategies to facilitate the uptake of the wheelchair skills training in pediatric rehabilitation settings.

Application of the Knowledge to Action (KTA) framework can optimize the translation of evidence into practice (14); in this case, the use of the WSTP in pediatric rehabilitation settings. According to the KTA framework, knowledge translation is a dynamic process that includes a knowledge creation funnel and knowledge-to-action phases. Adapting the knowledge to the local context is a critical action phase, which includes identifying required modifications to support pediatric considerations. Daoust et al. (2021) documented OTs' concerns about using the WSP in a pediatric setting, suggesting improvements were needed to address playfulness of the tools, skill choice, practicality, pediatric specificity, and resource constraints (10). These findings identified a need to develop clear, practical, and pediatric-friendly tools, which represents development of targeted knowledge translation tools and products in the knowledge creation funnel.

Three complementary knowledge transfer (KT) tools (i.e., storybook, instructional posters and training workbook) were developed by the members of our interdisciplinary research team (i.e., one adult wheelchair user, four researchers with expertise in wheelchair skills training, one researcher specialized in KT, two pediatric OTs, and 2 rehabilitation services managers). The purpose of this study was to describe OTs' and PMWUs' perceived satisfaction with the tools and to explore the perceived usability, relevance, and feasibility for meeting their needs.

\section{Methods}

\section{Study Design}

A descriptive qualitative study was conducted using a focus group (OTs) and interviews (PMWCs) following the Consolidated criteria for reporting qualitative research (COREQ) checklist (15). The study was approved by the Sainte-Justine University Hospital Research Center Ethics Board. OTs and PWMUs' parents provided informed consent and children provided assent.

\section{Participants}

Participants were recruited from the Marie Enfant Rehabilitation Center and its affiliated specialized elementary and high schools (i.e., VictorDoré and Joseph-Charbonneau schools) using a convenience sampling method. These three settings offer rehabilitation to children and teenagers up to 21 years old with physical disabilities in Montreal, Canada. OTs were recruited between July and September 2020 using an information letter and a narrated PowerPoint presentation sent by email. OTs were eligible to participate if they: 1) were currently employed as an OT at the pediatric rehabilitation center or one of its affiliated schools; 2) provided wheelchair skills training services; and 3) were able to participate in an online focus group in French. PMWUs were recruited between August and November 2020 using an invitation letter provided by their treating OT. PMWUs were eligible to participate if they: 1) were between 5 and 15 years old; 2) had received OT services at the pediatric rehabilitation center or one of its affiliated schools within the previous 3 months; 3 ) used a manual wheelchair at least 4 hours/day for at least the previous 6 months; 4 ) were able to understand simple instructions and participate in a French discussion online (i.e., by Zoom) for 60 minutes.

\section{Description of WSTP Knowledge Transfer Tools}

The tools addressed four indoor wheelchair skills: rolling forward and backward a short distance, turning while moving forward, and picking up an object from the floor. These skills are fundamental to learning more complex skills (e.g., ascend an incline, descend a curb) and are often required by PMWUs in school and community settings (e.g., moving in narrow hallways, picking up a pen, playing). The tools integrated some principles of motor learning (e.g., goal setting and monitoring, motivation, illustration, extrinsic feedback, and verbal cues). The tools were designed to be culturally and gender inclusive to reach diverse children (e.g., the characters had different skin colors and a gender-neutral physical appearance), using child-friendly language. Similar characters and illustrations were used in the three complementary tools to ensure continuity, as each tool was created for a specific purpose in the training process, but intended to be used as a package to facilitate engagement and learning.

Storybook. The storybook was developed to introduce wheelchair skills training in a stimulating and motivating way for PMWUs'. It was intended to create awareness among PMWUs, their family, friends and the multidisciplinary team, that children who use a manual wheelchair for mobility can learn wheelchair skills to be independent and engage in day-to-day activities. It featured Gab, a school-aged child, who goes on an adventure in the forest with his dog to discover a castle. The path to the castle is challenging and requires that Gab performs the four wheelchair skills.

Posters. Four instructional posters were created to provide visual support during training to help children understand the techniques required to perform the skills. They could also be used to provide reminders in a variety of settings (e.g., home, school, rehabilitation center). The 
skills were divided into smaller objective steps, which were illustrated in images. There were visual cues in the images to facilitate learning and integration.

Training Workbook. The training workbook, intended to be used collaboratively by the OT, child and parent, included three sections: 1) mobility goals, 2) skill progression, and 3) general comments. Children and OTs could draw smiley faces or add stickers on the workbook when they succeeded in each step.

\section{Procedures}

The WSTP KT tools were provided in advance to the participants to allow them to become familiar with them and thus facilitate the discussions. The focus group and semi-structured interviews were conducted using online videoconferencing (i.e., Zoom) to respect the COVID-19 public health restrictions. Sociodemographic information was collected from OTs (age, sex, work setting, years of experience, years working with PMWUs, frequency of wheelchair skills training; and use of the WSTP) and PMWUs (age, sex, years using a manual wheelchair, years using current wheelchair, previous wheelchair skills training, training location, assistance needed for wheelchair mobility). The semi-structured guides for the focus group and interviews were developed using the Guide to Monitoring and Evaluating Knowledge Management in Global Health Programs'indicators (16), which included: overall satisfaction (i.e., tools meet needs), usability (i.e., ease of use in terms of format [aesthetics], ease of understanding the content) and relevance (i.e., importance of content and applicability for OTs and PMWUs). The feasibility of implementing these KT tools into clinical practice was also documented (i.e., capacity to use the tools regarding time, environment, and personal characteristics).

Focus Group. A 90-minute focus group was conducted in French with OTs and co-facilitated by occupational therapy students (AAC, LFH, EL, IP). The focus group was pilot tested with the research team and modifications were made according to their suggestions to ensure the questions were clear and informed the objectives of the study. First, a brief background on the WSTP was provided and the KT tools were presented to set the stage for the discussion. Second, two thirty-minute periods of discussion were conducted, separated by a ten-minute break, to respond to eight open-ended questions and related probes. Examples of questions for each indicator included: "What is your first impression of these three tools?" (satisfaction), "What are your thoughts on the presentation and format of the tools?" (usability), "Are the skills explained and depicted appropriately, if not what modification would you make?" (relevance), and "In your current clinical context, is it feasible to use these tools?" (feasibility). In addition, three quantitative, closed questions were asked at the end of the focus group using the Zoom poll feature that captures anonymous data. Specifically, for each tool, OTs were asked to rate on a ten-point Likert Scale (1 [not at all] to 10 [totally]), if the tool would help them provide wheelchair skills training and if the format and aesthetics would facilitate wheelchair skills training. They were also asked to respond to a dichotomous question (i.e., yes or no) reporting their intention to use the tool in their clinical setting. A PowerPoint presentation was used as a visual support to facilitate the discussion. To make the process more interactive and engaging, OTs were given access to a Google Slides document (i.e., separate shared PowerPoint) where they could write short answers to the questions at times specified by the facilitators. The focus group was recorded.

Interviews. Sixty to ninety-minute interviews were conducted by an OT student (AAC) with PMWUs. According to the participants' preferences, a parent could accompany the PMWU during the interview to help them with Zoom, rephrase questions or clarify answers. First, rapport building involved introductions and general questions about PMWUs' interests. Then, PMWUs were asked seven open-ended questions and probes for the storybook, 12 for the posters and 14 for the training workbook. Examples of questions for each indicator included: "What do you like most and least about the tool?" (overall satisfaction), How do you like the characters? (usability), "Did the tool help you to learn new things for using your wheelchair?" (relevance). A PowerPoint presentation depicting the questions and tools was used as a visual support for the interview. As well, three smiley faces pictograms (i.e., happy, neutral, sad) were used to facilitate children's expression of their opinions regarding the tools. The interview process was pilot tested with a PMWU to ensure the questions were understandable for children and that they were able to express their points of view. It was modified according to his suggestions. The interviews were recorded.

\section{Data Analysis}

Descriptive statistics were calculated for the sociodemographic data (mean, percentages, standard deviations). The qualitative data were deductively analysed using the Framework method (17). Focus group and interview recordings were transcribed verbatim and anonymized. The focus group transcription was verified by randomly cross-checking $30 \mathrm{sec}$ increments with the recording and one interview transcription was entirely verified. Two matrices, one for the focus group (see Additional file 1) and one for the interviews (see Additional file 2), were collaboratively created by the research team and organized with the four indicators (overall satisfaction, usability, relevance, feasibility). The matrix for the interview was pre-tested with one interview and then refined. The focus group and five interview transcripts were independently coded by at least two team members. Even though mothers were not directly interviewed, the information they provided when assisting their children was coded and interpreted as it clarified PMWUs' answers and therefore allowed a more complete understanding of their points of

Page $4 / 13$ 
view. Coding discrepancies were resolved by discussion. The research team met to interpret the data. The direct quotes provided in this article have been translated from French to English by bilingual members of the research team. Quantitative focus group data were analysed using descriptive statistics. Percentages were calculated and data were visually illustrated in bar graphs.

\section{Results}

Eight OTs participated in the focus group (five from Victor-Doré primary school, one from Joseph-Charbonneau high-school and two from Marie Enfant Rehabilitation Center). Participants ranged from 26 to 54 years of age and had between 2 to 29 years of experience with PMWUs. Two OTs (25\%) reported rarely providing wheelchair skills training, five (62.5\%) said they did it occasionally, and one (12.5\%) reported doing so often. One OT (12.5\%) reported never using the WSTP for training, four OTs (50\%) rarely used it, two (25\%) OTs used it occasionally, and one OT (12.5\%) used it often for wheelchair skills training. Demographics information is presented in Table 1.

Table 1

Sociodemographic information of Occupational Therapists $(n=8)$

\begin{tabular}{|c|c|c|c|c|c|c|c|}
\hline Pseudonyms & $\begin{array}{l}\text { Age } \\
\text { (years) }\end{array}$ & Sex & $\begin{array}{l}\text { Experience at } \\
\text { current site } \\
\text { (years) }\end{array}$ & $\begin{array}{l}\text { Experience } \\
\text { with PMWUs } \\
\text { (years) }\end{array}$ & $\begin{array}{l}\text { Experience as } \\
\text { an OT (years) }\end{array}$ & $\begin{array}{l}\text { Frequency of } \\
\text { wheelchair skills } \\
\text { training }\end{array}$ & $\begin{array}{l}\text { Frequency } \\
\text { of WSTP } \\
\text { use }\end{array}$ \\
\hline & Mean=34.29 & & Mean=7.13 & Mean=8.13 & Mean=11.31 & & \\
\hline & $S D=10.63$ & & $S D=9.38$ & $S D=9.32$ & $S D=9.88$ & & \\
\hline Marie & 44 & Female & 6 & 6 & 22 & Often & Sometimes \\
\hline Audrey & 29 & Female & 2.5 & 2 & 6 & Rarely & Sometimes \\
\hline Alexandra & 28 & Female & 2 & 2.5 & 4.5 & Sometimes & Rarely \\
\hline Sara & NP & Female & 11 & 13 & 13 & Rarely & Rarely \\
\hline Nathalie & 32 & Female & 2.5 & 8.5 & 9 & Sometimes & Often \\
\hline Anna & 27 & Female & 2 & 2 & 3 & Sometimes & Rarely \\
\hline Kaitlyn & 26 & Female & 2 & 2 & 3 & Sometimes & Never \\
\hline Julie & 54 & Female & 29 & 29 & 30 & Sometimes & Rarely \\
\hline
\end{tabular}

Five PMWUs who received services from Marie Enfant Rehabilitation Center or an affiliated school were interviewed. Table 2 presents PMWUs' demographics. Participants were between six and 12 years old and had between three and nine and a half years of experience using a manual wheelchair. Two participants always or often needed help for wheelchair mobility and three sometimes needed help. Three PMWUs (60\%) previously received wheelchair skills training, two at school and one at the rehabilitation center. The mothers were partially or fully present during the interview. 
Table 2

Sociodemographic information of PMWUs $(n=5)$

\begin{tabular}{|c|c|c|c|c|c|c|c|c|c|c|c|c|}
\hline \multirow[t]{2}{*}{ Pseudonyms } & \multirow{2}{*}{$\begin{array}{l}\text { Age } \\
\text { (years) }\end{array}$} & \multirow[t]{2}{*}{ Sex } & \multirow{2}{*}{$\begin{array}{l}\text { Experience } \\
\text { using a } \\
\text { manual } \\
\text { WC } \\
\text { (years) }\end{array}$} & \multirow{2}{*}{$\begin{array}{l}\text { Experience } \\
\text { with } \\
\text { current WC } \\
\text { (years) }\end{array}$} & \multirow{2}{*}{$\begin{array}{l}\text { Previous } \\
\text { training }\end{array}$} & \multirow{2}{*}{$\begin{array}{l}\text { Frequency } \\
\text { of } \\
\text { assistance }\end{array}$} & \multicolumn{6}{|c|}{ Type of assistance } \\
\hline & & & & & & & S & VA & PAT & PAM & $\begin{array}{l}\text { PAM- } \\
0\end{array}$ & NP \\
\hline & Mean=9.60 & & Mean=6.30 & Mean $=2.40$ & & & & & & & & \\
\hline & $S D=2.61$ & & $S D=2.54$ & $S D=3.19$ & & & & & & & & \\
\hline Justine & 10 & Female & 6 & 1 & $\mathrm{RC}$ & Often & & & $x$ & & $x$ & \\
\hline Lucia & 6 & Female & 3 & 2 & No & Always & $x$ & $x$ & $x$ & $x$ & & \\
\hline James & 8 & Male & 5 & 0.5 & No & Sometimes & & & $x$ & $x$ & & \\
\hline Thomas & 12 & Male & 8 & 8 & School & Sometimes & $x$ & & $x$ & & $x$ & \\
\hline Catherine & 12 & Female & 9.5 & 0.5 & School & Sometimes & & & & & & $x$ \\
\hline
\end{tabular}

\section{Overall Satisfaction}

All OTs had positive first impressions of the three tools, describing them as "turnkey [Anna and Sara]", because they are ready to use in clinical settings to structure and deliver wheelchair skills training for PMWUs. They found the tools attractive, easy to use, playful, colorful, inclusive, and suitable. OTs emphasized that the KT tools would meet their wheelchair skills training needs as they may provide efficiencies in training, enhance children's engagement, and facilitate their knowledge. They also mentioned that the tools could be especially helpful for children with communication difficulties or intellectual disabilities and felt the tools could assist with transitions to wheelchair use.

PMWUs had mixed first impressions of the tools. Three PMWUs appreciated the tools, as they learned new wheelchair skills or improved their techniques for performing them. However, two PMWUs felt the tools were immature for their age and skills level. PMWUs particularly liked the dog, the castle, and the multicolored socks in the storybook, and found it funny and clear. However, they expressed the plot needed to be more exciting. For the posters, the "moving forward" skill was a favorite because of the rainbow used to clearly illustrate how the arms should move in an arc pattern. In the training workbook, PMWUs appreciated the table can be completed with their OT during training to track progress overtime and they particularly liked the idea of using smiley faces or stickers to highlight their success.

\section{Usability}

OTs considered that the format and aesthetics of all tools could facilitate the training of wheelchair skills (see Figure 1). Both OTs and PMWUs liked the colors, and OTs felt the use of primary colors provided good contrast. OTs appreciated the diversity of the characters (gender, nationality), suggesting that children connect more when resembling the characters. One PMWU described the characters as "cool [Justine]", appreciating that the characters were in a wheelchair like her. Regarding fonts, OTs felt the bold headings were easy to read, and liked that the selection of minimal yet effective words.

Some improvements were suggested to increase the usability of the tools. OTs suggested "to have a version that we could [...] personalize a bit to the needs of every child [Sara and Audrey]", essentially requesting modifiable versions of the tools to facilitate customization. OTs and PMWUs suggested that the illustrations of the wheelchair components should be more detailed and body positioning on the wheelchair could be depicted more accurately. For example, a PMWU stated "The hand rims of the wheelchair are missing. Usually, you push on them to move forward. Moreover, it isn't clear that Gab put his hand on his leg. Rather, it looks like he's trying to stop the wheelchair. His hand should be much closer to his thigh. [James]"

OTs appreciated the comprehensibility of the content, describing the language as suitable for young children and containing expressions frequently used in practice (e.g. "push, push, push", "yay"). They explained that the presence of images, the bright and clear signs, and the use of keywords made it easier to understand for PMWUs, especially for those with language difficulties. Despite these elements, PMWUs needed support to use the tools. Two PMWUs needed help to read the tools, especially for the storybook. Most PMWUs needed explanations to understand the purpose of the posters and training workbook, and the contexts in which they could be used. Three PMWUs had difficulties 
understanding the "Objectives" and "Levels" sections of the training workbook, suggesting to replace the word "Objectives" with "Challenges". One mother stated that these sections of the training workbook would be more relevant for OTs and parents than for children. A PWMU said: "Objectives make me uncomfortable. I don't want to have mobility objectives; I perceive my wheelchair as an asset and I want it to be fun. [Justine]". PMWUs needed explanations, demonstrations, or manual guidance for all tools to understand the different steps to perform the skills and the tips and tricks (e.g., the rainbow visual cue to show the movement of the arms for moving forward and backward). When understood, the tips and tricks were really appreciated by children: "Now that I understand why there is a rainbow on the wheel, I really like it [Justine]". To facilitate PMWUs' understanding, the same tips and tricks should be used in the tools (e.g., for the skill moving forward, the visual cue is an apple in the storybook and a rainbow in the posters). In addition, a small description of each step required to perform a skill should be added on the posters.

OTs and PMWUs liked the presentation, sequencing and progression to facilitate the acquisition of new wheelchair skills. When talking about the training workbook, a PMWU indicated: "the breakdown of each skill in small steps in the three levels gives children a lot of chances of success [James]". However, OTs suggested a more in-depth breakdown of each skill, as PMWUs with learning difficulties may need some extra steps to reach a skill level. In this regard, OTs and PWMUs proposed to add levels "performed with supervision and assistance [Kaitlyn]" to be inclusive of children who can't perform the skills independently. OTs and PMWUs proposed recommendations to facilitate the understanding of the skills steps (e.g., add a cloud and a sun on the rainbow to indicate where to put the hands on the rim at the beginning and at the end of the movement). They mentioned that the steps of the skill "Moving backward" were presented in a wrong order on the poster and proposed: "Look backward on the two sides should be the first step [James, Catherine, because if you move backward, hit someone and then look backward, it's not appropriate [James]".

\section{Relevance}

OTs indicated that the tools would help to provide wheelchair skills training to children (see Figure 2). In fact, they expressed interest and willingness to implement them into practice immediately, "as of tomorrow morning [Marie, Sara, Anna, Audrey and Nathalie]". The following quotes suggest intention and motivation for use: "it's already prepared and ready, and we only have to adapt it to the skill level of the child, I think it would be interesting to have it", "it is already much better than what I had done on my own, so it's something very interesting with which I can start [Marie]", and, "I was missing a bit of motivation to start, and time also. But now, [...] I have something concrete. I can start from that [Sara]". Several factors explained OTs' strong desire to use the tools, especially that the tools were adapted for young children. For example, an OT from Victor-Doré elementary school expressed: "They really target my clientele and are appropriate for my children's age, especially because of the drawings that are for kids. [Audrey]". OTs perceived possible time efficiencies and improved access to the WSTP, as the tools provide detailed information breaking the skills down for training in a pediatric-specific way. Furthermore, they believed the tools could increase PMWUs' engagement in the training process, as one OT explained when talking about the training workbook: "It's fun, centered around them and personalized. They can take ownership of their objectives and progress. Usually, we guide them more, but with this tool, they are more involved in the training process [Kaitlyn]."

Two PMWUs were motivated to practice wheelchair skills using the tools as they facilitated learning new skills through experience: "Before I received the tools, I never tried to pick an object from the floor. Now, I try new things with my wheelchair [Catherine]". They also mentioned having learned more efficient techniques that could foster their mobility and independence: "I learned that when I do big circles with my hands, like it is shown on the poster, I go faster. I can even move fast backward [Lucia]". One PMWU expressed the desire to use the tools because they gave good reminders of the appropriate techniques he sometimes forgets: "Sometimes you don't realize it, but instead of stopping the wheel and turning, you go fast and turn. Then you may hit the wall"[James]. The tools motivated him to change these habits he has adopted over time and to practice improving his wheelchair skills. In contrast, two PMWUs did not perceive the benefits of modifying their techniques and were not interested in using the tools, even though one had neck and back pain while performing certain skills. Nonetheless, these PWMUs stated that the tools could be useful for beginner PMWUs.

OTs and PMWUs perceived that the tools as less applicable to teenagers and experienced wheelchair users. The OT working at JosephCharbonneau High School felt the language, the presentation and the level of difficulty may be infantilizing for her clientele, except for PMWUs with intellectual disabilities. In this regard, when talking about the storybook, a ten-year-old PMWU stated: "It's good, but it's not appropriate for my age. [Justine]" Another PMWU mentioned: "I have been using a wheelchair for a very long time, but it is certain that those who have had one for a short time can learn things [Thomas]". Moreover, two mothers indicated that their children were "... more ready to learn how to go up sidewalk curbs, do wheelies and go downstairs. [mothers of Justine and James]". For the storybook, PMWUs suggested that explaining why Gab goes to the castle and modifying the plot to include more action would better capture their attention and make it more interesting, particularly for older children. OTs and PMWUs suggested we develop other KT tools focusing on higher-level skills and using a higher-level language for better engaging experienced wheelchair users and teenagers. 
OTs and mothers of PMWUs mentioned that other knowledge users could benefit from the tools, including but not limited to, other professionals involved in wheelchair provision (e.g., physiatrist, physiotherapists, social workers, psychologists), physical education teachers, family members and peers. For example, the physical education teacher may benefit from using the posters, as "She might be interested in having the posters to provide visual examples when practicing the skills with the children, to have them within her reach to support her teaching [Marie]". Moreover, the storybook could be used with non-PMWU peers to raise awareness about wheelchair use and capabilities of PMWU: "the storybook could be used at school to raise peers' awareness, so that they can understand that the wheelchair is like the child's legs [Justine's mother]".

Most OTs envisioned how the three tools could be used at different points in the wheelchair provision process. OTs, PMWUs and their mothers suggested distributing the storybook before or when they first receive their wheelchair, as it could help families cope with the transition and enable children to identify with people living in similar situations. OTs also suggested the storybook could be used to introduce the training process: "the story would be the introduction, the first thing to be presented to the child and their family [Kaitlyn]". OTs proposed two contexts for use of the posters, including on the school walls and at Marie Enfant Rehabilitation Center, as "a reminder that these tools exist and of the techniques to perform the skills [Kaitlyn]", and to "generalize the learning to different contexts [Kaitlyn]". OTs identified several applications for the training workbook, including during training as a "communication tool [Sara]", between OTs and parents or other team members, and to facilitate training at home. OTs perceived the tools could be used for individual and group interventions. In addition, an OT and a mother mentioned that the tools could be relevant "in many environments of a child's life [Anna]" and "at an early age", such as in kindergarten and at school.

\section{Feasibility}

The OTs felt optimistic about having the time to use the tools in their daily practice, as they target the elements of the WSTP relevant to PMWUs. One OT was confident they would be able to use the tool in the physical environment at Marie Enfant Rehabilitation Center: "We have the space to provide training. We have long hallways and a laboratory [Anna]". However, in an ideal world, OTs would have access to the same physical objects as presented in the tools to practice with PMWUs: "I would have real wood logs...I would have the material to put the story in a tangible form [Alexandra]".

Three PMWUs mentioned that the method proposed for the skill "Pick an object from the floor" was not feasible because their wheelchairs were too high. They suggested adding alternative methods for performing this skill safely (e.g., use long-handled reacher). A PMWU also had difficulties performing the skills using the method proposed in the tools due to difficulties with his left hand and back pain. These situations reinforced OT's suggestions of customizing tools for each child. Environmental barriers also limited PMWUs' opportunities to practice the skills using the tools. For example, one PMWU was not able to use his training workbook at home for practicing the skills because he lived in a small apartment. A mother mentioned that the winter season was also a limiting factor.

\section{Discussion}

In response to the need identified by Daoust et al. (2021), condensed, ready-to-use, child-friendly material was created to facilitate clinical use of the WSTP. Specifically, three KT tools were developed as a preliminary step to adapt the WSTP for pediatric clientele. This study evaluated OTs' and PMWUs' satisfaction and perspectives on usability, relevance, and feasibility of the three tools, with results demonstrating the tools have potential to foster the implementation of the WSTP in pediatric rehabilitation settings.

The inclusion of both OTs' and PMWUs' perspectives on the three tools provided in-depth feedback from the two primary end-users. Our findings highlight the critical importance of consulting both providers and users, especially children, in developing services that are aligned with their needs and preferences (18). OTs impressions influence whether or not the tools will be implemented in clinical practice, whereas PMWUs impressions will influence their level of engagement in the training sessions (19). Therefore, the current study aligns with the United Convention on the Rights of the Child (1989) and the Nothing About Us Without Us movement (1998) by supporting the rights of children to express their points of views on the decisions pertaining to them. Research incorporating the perspectives of children with disabilities regarding the interventions they receive are limited $(20,21)$.

In general, OTs and PMWUs provided positive feedback regarding the use of the tools with elementary school children and novice wheelchair users. All OTs expressed the desire to use the tools with their clients as soon as possible because they are easy to use in their context, may increase the efficiency of their interventions and make wheelchair skills training fun for children. Our findings demonstrate that the tools respond to the concerns regarding the playfulness of the WSTP, its practicality and its specificity in pediatrics, while decreasing the time barriers limiting its use (10). Three of five PMWUs perceived the tools could help them improve their mobility and were motivated to use them. The two PMWUs who felt they had already mastered the wheelchair skills (thus said they would not use the tools) were experienced wheelchair users who appeared to be ready to learn community and advanced skills. However, they did affirm that the tools could have 
benefits for younger children with less experience. Although this resonates with evidence supporting greater training effects in new wheelchair users (6), experienced children and adults have improved their wheelchair skills upon completion of the WSTP (5, 22). It is important to note that one PMWU had upper-body pain, a common wheelchair-related injury associated with overuse and poor propulsion techniques (23). Pediatric-onset wheelchair use is associated with activity-limiting pain in adulthood (23). Pain related to overuse might be prevented using more efficient techniques to perform wheelchair skills (23). As children may tend to have difficulties imagining themselves in the future and understanding the relationship between the techniques they use and pain, it might be useful to raise their awareness on the long-term benefits of wheelchair skills training.

OTs, PMWUs and mothers perceived that the tools could be used in a wide variety of contexts (e.g., home, kindergarten, school) and by diverse end-users (e.g., parents, peers, physical education teachers, physiatrist). This is particularly interesting given the need to increase children's opportunities for wheelchair skills training and the importance of starting training as early as possible (5, 24). As health professionals working in rehabilitation and school settings have numerous competing priorities, training others such as teachers or peers could alleviate some clinician burden. Wheelchair skills training could be delivered across the environments in which children live and grow (e.g., summer camps, sport activities, community organizations) to expose children to various environments and contexts in which their manual wheelchair may be used. For example, power wheelchair mobility training was offered within a specialized summer camp to five school-aged children with severe cerebral palsy $(25,26)$. After the camp, the impacts of the intervention extended beyond significatively improving the children's powered mobility skills to include positive changes in motor, cognitive, communication and social skills (25, 26).

Despite the recognized benefits of improved independent mobility on global development among children with disabilities' (27-29), parents are frequently reluctant to introduce wheelchair skills training early and commonly prioritize walking $(10,18,30)$. However, the literature does not indicate that wheelchair use limits the acquisition of walking skills (27). Moreover, children with mobility impairments frequently need a variety of mobility options, as walking may be difficult in certain circumstances (e.g., going shopping after school, long distances) (27). In fact, use of a wheelchair could limit fatigue and improve participation (e.g., wheel to the park and walk on the playground), thus reinforcing the importance of early familiarization with wheelchairs (27). In this regard, participants suggested the storybook could be distributed to families during wheelchair prescription to enhance acceptance and awareness about the increased independence children could gain by moving independently around their environments. Given the lack of such resources to support children and families during wheelchair procurement, the storybook may help to ease the transition (18). A precursor storybook may also help families to cope with the introduction to wheelchairs and could encourage wheelchair skills training. To date, such literature focuses mainly on power mobility (18). More evidence is needed to understand how PMWUs and their families could use a precursor storybook for manual wheelchairs.

The participants proposed several recommendations to improve the three WSTP KT tools. Adding more action to the plot, increasing playfulness, and writing two to three word-descriptions on the posters is relatively easy to modify. However, the suggestion to break the wheelchair skills into smaller steps (or add lower levels) in the training workbook to facilitate a level of success among all PMWUs may be harder to achieve. To date, there are no documented developmental milestones for manual wheelchair skills. Although the Wheelchair Skills Test classifies the wheelchair skills in order of difficulty (4), a developmental sequence of wheelchair skills acquisition could be helpful for pediatric clinicians with concerns about skill choice (10). A systematic review of pediatric occupational therapy interventions demonstrated that a common characteristic shared by the most effective interventions for improving motor skills is a scaffolding approach (i.e., breaking the skills down in small steps) (31). The scaffolding approach is one of the motor learning principles included in the WSTP (4), but more research is needed for pediatric wheelchair skills to provide a 'just-right challenge' and allow children to experience success throughout the learning process (10).

Other recommendations for improving the tools included improving the illustrations depicting how to perform the wheelchair skills, standardizing the visual cues in all three tools and rendering the tools available in formats to allow OTs to customize them to the specific needs of their clients. Electronic fillable formats also facilitate communication and collaboration with parents, since OTs frequently use emails to follow-up with them (10). Posters for adults are already available in PDF on the WSP website (32) and the WSP manual encourages clinicians to make adaptations to meet their clients' needs $(4,10)$. The WSP website appears to be a strategic platform to diffuse the learning resources; as it has been accessed by 145,381 users from 193 countries and the WSP YouTube channel had 141,200 views up to June 17, 2021 (32). Following the suggestions of OTs and PMWUs, the tools developed in the current studies will be refined and additional tools addressing skills at the community and advanced levels will be developed. Finally, as a last step, the tools will be pilot tested by OTs with PMWUs.

\section{Limitations}

Although our study was limited by a small sample size, the inclusion of both OTs and PMWUs' provided in-depth feedback from two primary end-users (including children with disabilities who are often overlooked stakeholders in research). Despite the strategies used to elicit 
PMWU's perceptions of the tools (e.g., PowerPoint visual support, pictograms, parental assistance), challenges arose when conducting the interviews that may have affected our results. PMWUs' responses tended to be brief, and it was sometimes difficult to gain deeper insights on their perspectives (e.g., understand why they don't want to use the tools). It was also difficult to maintain PMWU's attention, concentration, and motivation to respond to the questions, which may be explained by several factors. The interviews were conducted by a student who had limited experience in collecting qualitative data with children. Moreover, children may have experienced fatigue during 60 and 90 minutes, or may have been distracted (e.g., by toys), potentially prompting parents to respond in their place. Parents sometimes interfered by distracting the PMWUs (e.g., laughing) or suggesting answers that could influence the child's opinion. To prevent this, we could have obtained parents' feedback on the tools before interviewing the children or provided ground rules regarding their participation.

Finally, the tools were provided in advance to PMWUs rather than seeing them on the screen for the first time. Although most PMWUs had time to review the tools with their parents prior to the interview, they may have had difficulties with recall, forgetting their first impressions on viewing them. Moreover, we observed that parents often described the tools to their children, which may have limited our ability to elicit their first impressions of them. It may have been more useful to introduce the tools on the call, and yet, sharing the tools in advance allowed PMWUs to try them out.

\section{Conclusions}

OTs and PMWUs were satisfied with the WSTP KT tools, perceiving them to be usable, relevant, and feasible for use in clinical practice. These tools represent a concrete solution to address the gap between the availability of the Wheelchair Skills Training Program and its use in pediatric rehabilitation settings. Additional KT tools that focus on intermediate and advanced skills are needed to cover the full range of WSTP skills and reach children of different ages and skill levels and should be developed using a similar process whereby feedback is sought from end-users. user-centered process.

\section{List Of Abbreviations}

OT(s): Occupational therapist(s)

PMWU(s): Pediatric manual wheelchair user(s)

WSP: Wheelchair Skills Program

WSTP: Wheelchair Skills Training Program

KTA: Knowledge to action

KT: Knowledge transfer

\section{Declarations}

Ethic Approval and Consent to Participate: The study was approved by the Sainte-Justine University Hospital Research Center Ethics Board (\#2020-2828). OTs and PWMUs' parents provided written informed consent in accordance with the Declaration of Helsinki and the guidelines of the Tri-Council Policy Statement: Ethical Conduct for Research Involving Humans (2018). Parents were encouraged to obtain children's assent to participate.

Consent for Publication: Not applicable

Availability of data and materials: The data generated and analysed in the study cannot be shared to respect participants' confidentiality. The anonymous datasets forms are available from the corresponding author on reasonable request. The study materials to analyse the data are available in additional files.

Standards of reporting: Authors performed all methods and reported them in accordance with the relevant guidelines (i.e., COREQ) and regulations.

Competing interests: The authors declare that they have no competing interests.

Funding: This project was supported by the Child-Bright Network. The funder was not involved in the study development and conduct, neither it was involved in the analysis and interpretation of data, writing of the report, or in the decision to submit the article for publication. 
Authors' contributions: PR and KB conceptualized the study and protocol and were primarily responsible for the development and coordination of the study. AAC, LFH, EL and IP developed the 3 KT tools and collected data. MR, FR, MB, PR and KB provided feedback on the tool prototypes. BO, AAC, LFH, EL, IP, MR, FR, YBM, PR and KB contributed to data interpretation and analysis. BO led the manuscript. All authors have reviewed and approved the final manuscript.

Acknowledgements: The authors thank the participants for sharing their perspectives.

\section{References}

1. McConachie H, Colver AF, Forsyth RJ, Jarvis SN, Parkinson KN. Participation of disabled children: how should it be characterised and measured? Disabil Rehabil. 2006;28(18):1157-64.

2. Rodby-Bousquet E, Hägglund G. Use of manual and powered wheelchair in children with cerebral palsy: a cross-sectional study. BMC Pediatr. 2010;10(1):59.

3. Huhn K, Guarrera-Bowlby P, Deutsch JE. The Clinical Decision-Making Process of Prescribing Power Mobility for a Child with Cerebral Palsy. Pediatr Phys Ther. 2007;19(3):254-60.

4. Kirby RL, Rushton PW, Smith C, Routhier F, Archambault PS, Axelson PW, et al. Wheelchair Skills Program Manual: Version 5.2. 2021. https://wheelchairskillsprogram.ca/wp-content/uploads/WSP-Manual-version-5.2.1.approved-version.pdf. Accessed Aug 302021.

5. Sawatzky B, Rushton PW, Denison I, McDonald R. Wheelchair skills training programme for children: a pilot study: acquisition of wheelchair skills in children. Aust Occup Ther J. 2012;59(1):2-9.

6. Keeler L, Kirby RL, Parker K, McLean KD, Hayden JA. Effectiveness of the Wheelchair Skills Training Program: a systematic review and meta-analysis. Disabil Rehabil Assist Technol. 2019;14(4):391-409.

7. Tu C-J, Liu L, Wang W, Du H-P, Wang Y-M, Xu Y-B, et al. Effectiveness and safety of wheelchair skills training program in improving the wheelchair skills capacity: a systematic review. Clin Rehabil. 2017;31(12):1573-82.

8. Wilson D, Best KL, Miller WC. Peer-led approaches for improving satisfaction with participation, wheelchair skills and self-efficacy among children who use manual and power wheelchairs. In International Seating Symposium Mar 5 2020. Vancouver: Canada; 2020.

9. Daoust G, Rushton PW, Demers L. Bridging the gap in wheelchair skills testing and training in a Canadian paediatric rehabilitation context. In Oceania Seating Symposium Nov 2013 2019. Melbourne: Australia; 2019.

10. Daoust G, Rushton PW, Racine M, Leduc K, Assila N, Demers L. Adapting the Wheelchair Skills Program for pediatric rehabilitation: recommendations from key stakeholders. BMC Pediatr. 2021;21(1):103.

11. Best KL, Routhier F, Miller WC. A description of manual wheelchair skills training: current practices in Canadian rehabilitation centers. Disabil Rehabil Assist Technol. 2015;10(5):393-400.

12. Bloemen MA, Verschuren O, van Mechelen C, Borst HE, de Leeuw AJ, van der Hoef M, et al. Personal and environmental factors to consider when aiming to improve participation in physical activity in children with Spina Bifida: a qualitative study. BMC Neurol. 2015;15(1):11.

13. Barnard AM, Nelson NG, Xiang H, McKenzie LB. Pediatric Mobility Aid-Related Injuries Treated in US Emergency Departments From 1991 to 2008. Pediatrics. 2010;125(6):1200-7.

14. Graham ID, Logan J, Harrison MB, Straus SE, Tetroe J, Caswell W, et al. Lost in knowledge translation: Time for a map? J Contin Educ Health Prof. 2006;26(1):13-24.

15. Tong A, Sainsbury P, Craig J. Consolidated criteria for reporting qualitative research (COREQ): a 32-item checklist for interviews and focus groups. Int J Qual Health Care. 2007;19(6):349-57.

16. Ohkubo S, Sullivan TM, Harlan SV, Timmons BK, Strachan M. Guide to monitoring and evaluating knowledge management in global health programs. Ward Rinehart. 2013. https://www.msh.org/sites/default/files/km-monitoring-and-eval-guide.pdf. Accessed 30 Aug 2021.

17. Gale NK, Heath G, Cameron E, Rashid S, Redwood S. Using the Framework method for the analysis of qualitative data in multidisciplinary health research. BMC Med Res Methodol. 2013;13(1):117.

18. Bray N, Noyes J, Edwards RT, Harris N. Wheelchair interventions, services and provision for disabled children: a mixed-method systematic review and conceptual framework. BMC Health Serv Res. 2014;14(1):309.

19. King G, Chiarello LA, Ideishi R, D'Arrigo R, Smart E, Ziviani J, et al. The Nature, Value, and Experience of Engagement in Pediatric Rehabilitation: Perspectives of Youth, Caregivers, and Service Providers. Dev Neurorehabilitation. 2020;23(1):18-30.

20. Bailey S, Boddy K, Briscoe S, Morris C. Involving disabled children and young people as partners in research: a systematic review: Involving disabled children and young people as partners in research. Child Care Health Dev. 2015;41(4):505-14.

Page 11/13 
21. Wickenden M, Kembhavi-Tam G. Ask us too! Doing participatory research with disabled children in the global south. Childhood. 2014;21(3):400-17.

22. Best KL, Miller WC, Huston G, Routhier F, Eng JJ. Pilot Study of a Peer-Led Wheelchair Training Program to Improve Self-Efficacy Using a Manual Wheelchair: A Randomized Controlled Trial. Arch Phys Med Rehabil. 2016;97(1):37-44.

23. Hwang M, Zebracki K, Chlan KM, Vogel LC. Longitudinal changes in medical complications in adults with pediatric-onset spinal cord injury. J Spinal Cord Med. 2014;37(2):171-8.

24. Huegel M, Otieno S, Kenyon LK. Validity of the WST and the WST-Q in children with spina bifida: a pilot project. Disabil Rehabil Assist Technol. 2019;14(7):744-50.

25. Rosenberg L, Maeir A, Gilboa Y. Feasibility Study of a Therapeutic Mobility Summer Camp for Children with Severe Cerebral Palsy: Power Fun. Phys Occup Ther Pediatr. 2020;40(4):395-409.

26. Rosenberg L, Cohen R, Maeir A, Gilboa Y. Effects of a powered mobility summer camp as perceived by school staff: a qualitative study. Disabil Rehabil Assist Technol. 2021;1-8.

27. Livingstone R, Paleg G. Practice considerations for the introduction and use of power mobility for children. Dev Med CHILD Neurol. 2014;56(3):210-21.

28. Livingstone R, Field D. Systematic review of power mobility outcomes for infants, children and adolescents with mobility limitations. Clin Rehabil. 2014;28(10):954-64.

29. Rousseau-Harrison K, Rochette A. Impacts of wheelchair acquisition on children from a person-occupation-environment interactional perspective. Disabil Rehabil Assist Technol. 2013;8(1):1-10.

30. Pituch E, Rushton PW, Ngo M, Heales J, Poulin Arguin A. Powerful or Powerless? Children's, Parents', and Occupational Therapists' Perceptions of Powered Mobility. Phys Occup Ther Pediatr. 2019;39(3):276-91.

31. Novak I, Honan I. Effectiveness of paediatric occupational therapy for children with disabilities: A systematic review. Aust Occup Ther J. 2019;66(3):258-73.

32. Dalhousie University. Wheelchair Skills Program. https://wheelchairskillsprogram.ca/en/ (2021). Accessed 1 Aug 2021.

\section{Figures}

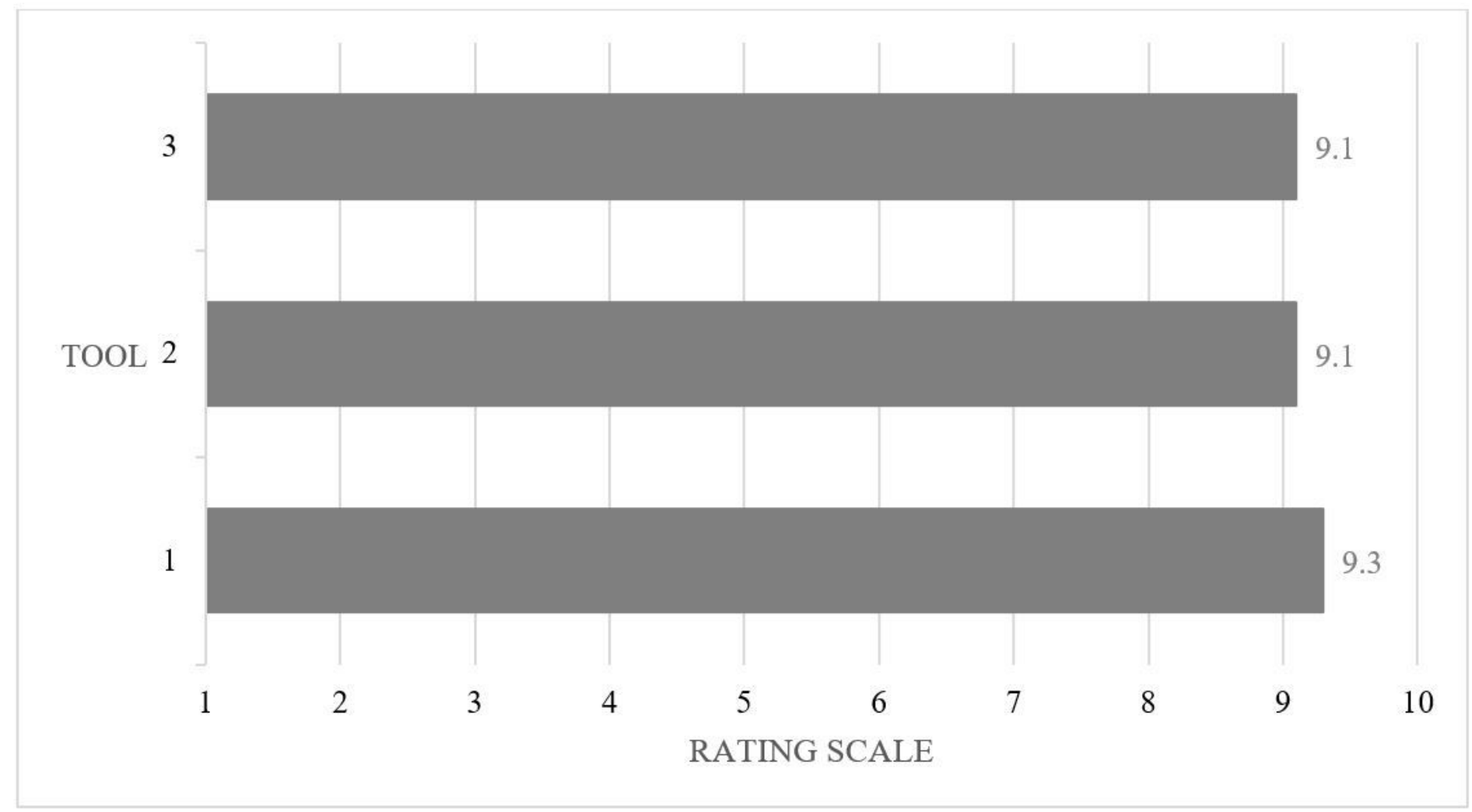


OTs' perceptions: format and aesthetics of the tools could facilitate the training of wheelchair skills

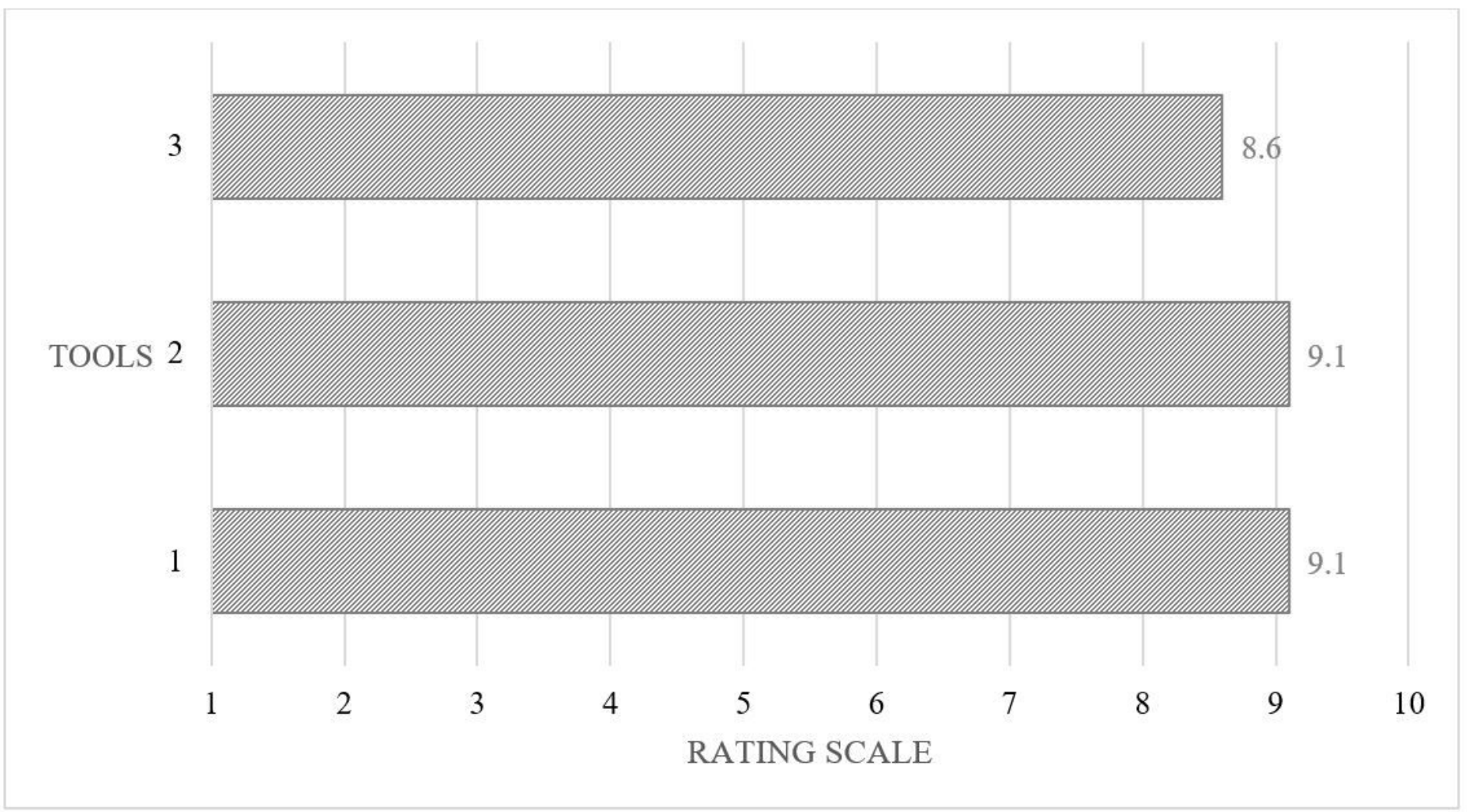

\section{Figure 2}

OTs perceptions on the tools' relevance to help them in the training of children's wheelchair skills

\section{Supplementary Files}

This is a list of supplementary files associated with this preprint. Click to download.

- Additionalfile1.docx

- Additionalfile2.docx 